\title{
Satisfiability by Maxwell-Boltzmann and Bose-Einstein Statistical Distributions
}

CLAUDIO ANGIONE, Computer Laboratory, University of Cambridge, UK

ANNALISA OCCHIPINTI, Computer Laboratory, University of Cambridge, UK

GIUSEPPE NICOSIA, Department of Mathematics and Computer Science - University of Catania and Institute for

Advanced Studies, Italy

Recent studies in theoretical computer science have exploited new algorithms and methodologies based on statistical physics for investigating the structure and the properties of the Satisfiability (SAT) problem. We propose a characterization of the SAT problem as a physical system, using both quantum and classical statistical-physical models. We associate a graph to a SAT instance and we prove that a Bose-Einstein condensation occurs in the instance with higher probability if the quantum distribution is adopted in the generation of the graph. Conversely, the fit-get-rich behavior is more likely if we adopt the Maxwell-Boltzmann distribution. Our method allows a comprehensive analysis of the SAT problem based on a new definition of entropy of an instance, without requiring the computation of its truth assignments. The entropy of a SAT instance increases in the satisfiability region as the number of free variables in the instance increases. Finally, we develop six new solvers for the MaxSAT problem based on quantum and classical statistical distributions, and we test them on random SAT instances, with competitive results. We experimentally prove that the performance of the solvers based on the two distributions depends on the criterion used to flag clauses as satisfied in the SAT solving process.

Categories and Subject Descriptors: F.2.0 [Analysis of algorithms and problem complexity]: General

General Terms: Design, Algorithms, Performance, Theory

Additional Key Words and Phrases: SAT, Complex network, Phase transition, Maxwell-Boltzmann distribution, BoseEinstein distribution, SAT solvers, quantum and classical system, Physics-inspired computation

\section{INTRODUCTION}

Nowadays, the SAT problem is regularly used for solving large-scale computational problems, such as AI planning, protein structure prediction, haplotype inference, circuit-level prediction of crosstalk noise, model checking, and hardware and software verification [Clarke et al. 2008]. As a result, it has received significant research attention [Du et al. 1997; Mézard et al. 2002], and numerous solver algorithms have been proposed and improved (e.g., by analyzing the structure of each instance [Hamadi et al. 2009]). Since evaluating all these techniques requires the generation of hard satisfiable instances [Spence 2010], several methods for generating random instances have been presented to test the solvers performance [SAT-competition 2013].

Many SAT solvers are based on local search techniques [Grégoire et al. 2009], linear programming [Zimmermann and Monfroglio 1997], or tabu search [Nonobe and Ibaraki 1998]. New methods for the SAT problem analysis consist of translating each instance into a graph (e.g., by using the planar graph approach [Kautz and Selman 1999] or the Bose-Einstein distribution and the S2G algorithm based on quantum physics [Angione et al. 2013]). On the other hand, over the last few years, several research fields have witnessed a remarkable expansion due to the collaboration between physicists and computer scientists [Mitchell et al. 1992; Mézard and Montanari 2007; Mézard and Mora 2009]. Therefore, studying the $k$-SAT problem through models used in statistical physics represents an unprecedented opportunity to find a new characterization for the satisfiability problem.

In this work, we prove that the $\mathrm{S} 2 \mathrm{G}$ algorithm is unlikely to generate Bose-Einstein condensed graphs when using the Maxwell-Boltzmann distribution (i.e., classical physics instead of quantum physics). To this aim, we propose a version of the S2G algorithm based on the Maxwell-Boltzmann distribution and we produce its phase diagrams, which we compare to the Bose-Einstein case. We also define the entropy of a $k$-SAT instance and we introduce its temperature, in order to translate each instance into a fully characterized physical system (summarized in Table I). Bose-Einstein graphs have lower entropy than Maxwell-Boltzmann ones, in agreement with the physical distribution of particles. In the phase diagram of a SAT formula, high entropy is associated with many free variables available to satisfy the few clauses of the formula, which is a typical feature of the SAT 
Table I. Dictionary translating the graph (left) into the $k$-SAT problem (center) and statistical physics language (right)

\begin{tabular}{|c|c|c|}
\hline$G=(V, E)$ & $k$-SAT & Statistical physics \\
\hline node & clause & degeneration state of the energy level of the node \\
\hline edge & link between two clauses & one particle for each degeneration state involved \\
\hline node weight & fitness of a clause & value of the energy level \\
\hline edge weight & probability of being established & weight on particles \\
\hline out-degree update & parameter $\theta$ & temperature of the system \\
\hline
\end{tabular}

phase. Finally, we propose six SAT solvers inspired by CHAINSAT [Alava et al. 2008] and PROBSAT [Balint and Fröhlich 2010] and augmented with classical and quantum statistical-physical approaches to classify a SAT instance. The computational effort and the number of clauses satisfied by the algorithm depend on the physical parameters (classical or quantum) and on the criterion chosen to satisfy the instances in CHAINSAT and in PROBSAT.

The remainder of the paper is organized as follows. In Section 2, we introduce the Bose-Einstein distribution. Section 3 describes the Maxwell-Boltzmann distribution and the S2G version based on the classical physics. In Section 4, we define the entropy of a $k-$ SAT instance. Fnally, we describe the six new SAT solvers in Sections 5 and 6.

\section{A CHARACTERIZATION OF SAT THROUGH QUANTUM PHYSICS}

By translating a SAT formula into a graph, the $S 2 \mathrm{G}$ algorithm [Angione et al. 2013] shows evidence of a process equivalent to the Bose-Einstein condensation in quantum physics. A vertex $v_{i}$ is a clause $C_{i}$ of the $k$-SAT formula $F=C_{1} \wedge C_{2} \wedge \ldots \wedge C_{m}$. The graph is built by defining global and local fitness functions (named $f^{G}$ and $f^{L}$ respectively) for evaluating literals and clauses. Then, a metric is defined to compute how many literals are not in common between two clauses. This metric is proved to be related to the Hamming distance [Richardson and Urbanke 2008]. At each iteration of S2G, a new clause of the SAT formula is added to the graph as a node, and its energy is computed. Then, the clause is linked to a clause already in the graph, and a particle is put on both the energy levels of the clauses sharing the link.

\subsection{The Bose-Einstein distribution}

Let us consider an isolated system of $N$ identical and indistinguishable bosons confined to a space of volume $V$ and sharing a given energy $E$. Let us assume that these bosons can be distributed into a set of energy levels, where each level $E_{i}$ is characterized by an energy $\epsilon_{i}$ and a degeneration $g_{i}$, representing the number of different physical states that can be found at that level. The $N$ identical and indistinguishable particles are distributed among the energy levels, and each level $E_{i}$ contains $n_{i}$ particles, to be accommodated among its $g_{i}$ quantum states. One can readily check that $n_{i}$ particles may be put on the level $E_{i}$ (consisting of $g_{i}$ states) in $\left[n_{i}+\left(g_{i}-1\right)\right]$ ! different ways. Since bosons are indistinguishable and the physical states are equivalent, the number of possible assignments of $n_{i}$ bosons on $E_{i}$ is $w_{i}=\frac{\left(n_{i}+g_{i}-1\right) !}{n_{i} !\left(g_{i}-1\right) !}=\left(\begin{array}{c}n_{i}+g_{i}-1 \\ n_{i}\end{array}\right)$. By iterating for all the energy levels $E_{i}$, a distribution $\left\{n_{i}\right\}$ (i.e., a distribution with $n_{i}$ particles on the level $E_{i}, \forall i$ ) can be obtained in $W=\prod_{i} w_{i}$ different ways. Specifically, $w_{i}$ is the number of distinct microstates associated with the $i$ th level of the spectrum, while $W$ is the number of distinct microstates associated with the whole distribution set $\left\{n_{i}\right\}$.

The distribution corresponding to the statistical equilibrium is the most probable one, thus it is the one that may be reached in the largest number of possible ways. Hence, we compute the maximum $W$ subject to the conservation of the number of particles $\sum_{i} n_{i}=N$ and to the preservation of the energy of the system $\sum_{i} \epsilon_{i} n_{i}=E$. Using the method of Lagrange's multipliers (applied to $\log W$ ), we have the following definition of Bose-Einstein distribution:

$$
n_{i}=\frac{g_{i}}{e^{\alpha+\beta \epsilon_{i}}-1},
$$


where $\alpha=-\frac{\mu_{C}}{k_{B} T}$ and $\beta=\frac{1}{k_{B} T}$ are inversely proportional (by means of Boltzmann's constant $k_{B}$ ) to the absolute temperature $T$ of the system at the equilibrium, and $\mu_{C}$ represents the chemical potential [Pathria 1996].

Given an ideal Bose-Einstein gas in equilibrium below its transition temperature, the BoseEinstein Condensation (BEC) is the property that a finite fraction of particles occupies the lowest energy level. Below a critical temperature $T_{B E C}$ near to $0 \mathrm{~K}$, all the particles become absolutely identical, with no possible measurement that can tell them apart. The gas shows a very unusual state of aggregation of particles, called Bose-Einstein condensate, also referred to as "the fifth state of matter". According to Penrose and Onsager [Penrose and Onsager 1956], we can provide a criterion of $\mathrm{BEC}$ for an ideal gas in equilibrium: $\mathrm{BEC} \Longleftrightarrow \frac{\left\langle n_{0}\right\rangle}{N}=e^{O(1)}$, No BEC $\Longleftrightarrow \frac{\left\langle n_{0}\right\rangle}{N}=o(1)$, where $\left\langle n_{0}\right\rangle$ is the average number of particles that occupy the lowest energy level $E_{0}$.

\subsection{Condensation phenomena in a SAT formula}

The graph construction is thought of as a dynamical process. Given the graph $G=(V, E)$ at the $(i-1)$ th iteration, the $\mathrm{S} 2 \mathrm{G}$ algorithm adds a clause $C_{t_{i}}$ to $G$ as a node $v\left(C_{t_{i}}\right)$, by estimating the probability of being connected to each node $v\left(C_{t_{j}}\right)$ already in the graph as

$$
\Pi_{t_{j}}=\frac{k_{t_{j}} \cdot f^{L}\left(C_{t_{j}}\right)}{\sum_{\nu=1}^{|V|} k_{t_{\nu}} \cdot f^{L}\left(C_{t_{\nu}}\right)},
$$

where $k_{t_{j}}=\operatorname{degree}\left(v\left(C_{t_{j}}\right)\right)$ is the connectivity of $C_{t_{j}}$ (i.e., the number of links shared by $v\left(C_{t_{j}}\right)$ ), and $f^{L}\left(C_{t_{j}}\right)$ is the fitness of the clause $C_{t_{j}}$. This probability distribution ensures that a new vertex is likely linked to an existing one with high fitness value or/and high connectivity [Bianconi and Barabási 2001]. A node $v\left(C_{t_{i}}\right)$ entering the graph is assigned the energy

$$
\epsilon_{t_{i}}=-T \cdot \log f_{r}^{L}\left(C_{t_{i}}\right),
$$

where $T=1 / \beta$, and $\beta$ is a parameter used to model the temperature of the system.

In the mapping of $\mathrm{S} 2 \mathrm{G}$ to quantum physics, every clause of the graph is associated with the degeneration state of the energy level of that clause. For each link established between two clauses, the S2G algorithm assigns a particle to each of the two degeneration states of the two clauses involved. The S2G-PA version of the algorithm also includes the concept of preferential attachment. In particular, at each iteration $i$, the node that joins the graph is forced to connect to a number of nodes between 1 and a fixed upper bound $\rho$. Furthermore, an outgoing link is rewarded $\theta<1$, whereas an incoming link is rewarded 1, and therefore the graph is regarded as a directed graph. The condensation of the SAT formula over its fittest clause is eventually mapped to the emergence of a star-like topology in the graph. This phenomenon is associated with the BEC of bosons on the lowest energy level available.

\section{THE MAXWELL-BOLTZMANN S2G}

An approach to construct graph from $k$-SAT instances may also involve classical physics, namely the Maxwell-Boltzmann (MB) distribution. Here we address the key question whether the S2G algorithm [Angione et al. 2013] could generate Bose-Einstein (BE) condensed graphs by using the MB distribution. In particular, we prove that the BEC in the satisfiability problem is more likely to occur if we use the BE distribution in the construction of the graph. To this end, we develop a new version of the $\mathrm{S} 2 \mathrm{G}$ algorithm to generate graphs based on the $\mathrm{MB}$ distribution and analyze their phase diagrams, comparing the behavior in the $\mathrm{MB}$ and $\mathrm{BE}$ cases.

\subsection{The Maxwell-Boltzmann distribution}

From the classical-physics standpoint, particles must be regarded as distinguishable entities. The $N$ particles are distributed among the energy levels, and each level $E_{i}$ contains $n_{i}$ particles. Note that in the MB case the set $\left\{n_{i}\right\}$ of occupation numbers does not fully describe the system, since particle 


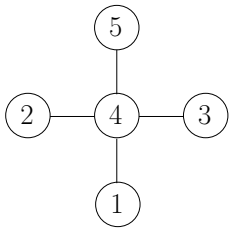

(a) BE case

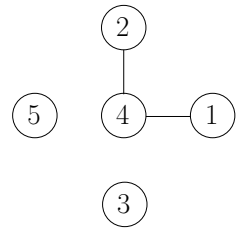

(b) MB case

Fig. 1. Different graphs with the same $F W=1$. In (a), a BEC has taken place (i.e., one node has a huge fraction of edges and the remaining fraction is shared among all the other nodes), while (b) does not show any condensation.

are distinguishable. Therefore, we need to distinguish between which particles are occupying each energy level. In other words, we need to count not merely the possible sets $\left\{\left\{n_{i}\right\}_{i}\right\}$ of occupation numbers, but also the possible microstates in each set.

The number of possible sets of occupation numbers is counted by taking into account that particles are distinguishable. Therefore, the selection of which particle is accommodated on which energy level must be ordered. At this stage, we do not investigate the order inside energy levels because we will do so when counting the microstates. As a result, the distribution set $n_{i}$ is obtainable in $\frac{N !}{\prod_{i} n_{i} !}$ different ways. Nevertheless, due to the Gibbs correction factor (see [Pathria 1996]) or, equivalently, using Stirling's approximations for the factorial $N$ !, we obtain the correct counting $\frac{1}{\prod_{i} n_{i} !}$.

As regards microstates, since particle are distinguishable, when accommodating $n_{i}$ particles on the energy level $E_{i}$, any of its $n_{i}$ particles may be put into any of its $g_{i}$ quantum states independently from one another, and all the resulting microstates are considered as distinct. In other words, each particle must be assigned one of the $g_{i}$ quantum states, and this can be done in $g_{i}$ different ways. By iterating this assignment independently from one particle to another, $n_{i}$ particles may be put on the level $E_{i}$ (consisting of $g_{i}$ states) in $g_{i}^{n_{i}}$ different ways.

Combining the two results, we finally obtain $W=\prod_{i} \frac{g_{i}^{n_{i}}}{n_{i} !}$, which is the total number of possible distinct microstates of the system. Using the same method as in the BE case, this expression leads to the following definition of Maxwell-Boltzmann distribution:

$$
n_{i}=\frac{g_{i}}{e^{\alpha+\beta \epsilon_{i}}}
$$

\subsection{A S2G version based on classical physics}

Since particles in S2G algorithm are associated with links between clauses in the graph of a SAT instance, and since the particles of the MB distribution are distinguishable, the idea underlying the MB version of the $\mathrm{S} 2 \mathrm{G}$ algorithm is that also the links between clauses must be distinguishable. Therefore, when we run the S2G algorithm using the MB distribution, we (i) switch off the preferential attachment (PA) used in [Angione et al. 2013], and (ii) ensure that if a new node $i$ will be linked to a node $j$ with probability $p_{i j}$, then $\operatorname{degree}(i)=\operatorname{degree}(i)+p_{i j}$ and $\operatorname{degree}(j)=\operatorname{degree}(j)+p_{i j}$. This new pattern permits that each new node is connected to a variable number of nodes in the graph (including zero), and therefore could generate graphs with many connected components. Hence, the original definition of Fraction Winner

$$
F W=w_{\text {links }} / t_{\text {links }},
$$

where $w_{\text {links }}=$ number of links shared by the winner, and $t_{\text {links }}=$ number of links of the whole graph [Angione et al. 2013], leads to graphs with the same FW, but with different topological structure (Figure 1).

Our goal is to study whether the graph built by using the MB distribution can show cases of BEC. For this reason, we introduce a new definition of $\mathrm{FW}$, which takes into account the presence of connected components. Let $G(V, E)$ be a graph with $|V|$ vertices and $|E|$ edges. We compute 


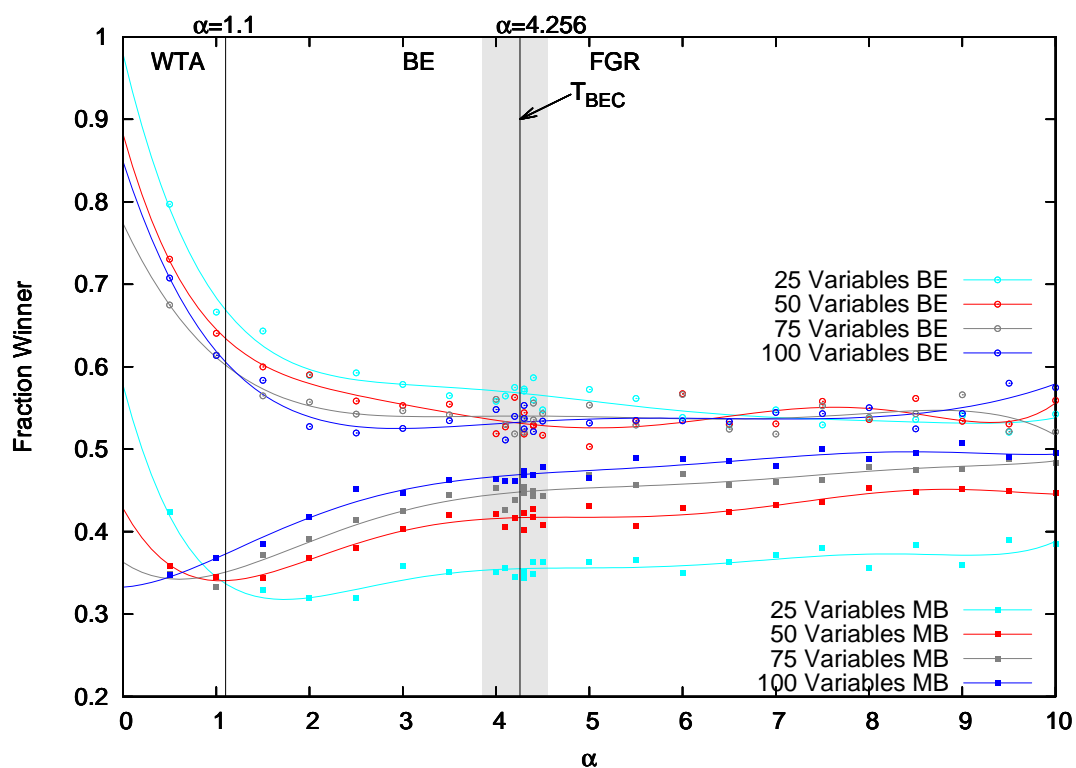

Fig. 2. Generalized Fraction Winner BE-MB. This plot shows the comparison between the GFW in the MB and BE cases. The MB plots always shows lower values than BE plots. Hence, BEC takes place under BE conditions with more probability than under MB conditions. The experimental data points have been fitted with a sixth-order regression curve using the leastsquares approach. The gray box shows the region of the phase diagram where the phase transition between satisfiable and unsatisfiable instances of 3-SAT is located [Monasson et al. 1999; Achlioptas and Ricci-Tersenghi 2009].

the number of connected components (CCs) by running the Depth First Search (DFS) algorithm [Cormen et al. 2001]. From the CCs, we obtain the number of nodes shared by these components (CNs), namely all the nodes of $G$ with nonzero degree. Then, we define the Generalized Fraction Winner as

$$
G F W=F W \cdot C N s /|V| .
$$

We remark that this definition is a generalization of the previous definition of FW. Indeed, in the BE construction, the PA ensures that CNs $=|V|$. In Figure 2 we show the comparison between the GFW in the $\mathrm{BE}$ and $\mathrm{MB}$ cases. Remarkably, the MB values are always lower than $\mathrm{BE}$ values, indicating that the probability of Bose-Einstein condensation is higher if the BE distribution is adopted in the S2G algorithm.

\section{EXPERIMENTAL RESULTS}

All the results included in this section have been obtained with an experimental protocol consisting of 100 SAT instances for each combination of parameter values, with 30 S2G graph generations per instance.

\subsection{The entropy of a $k$-SAT instance}

The aim of this section is to characterize the $k$-SAT problem as a physical system, in order to analyze the complexity of certifying the satisfiability of a random $k$-SAT instance, and eventually to find a satisfying assignment. Let us introduce a definition of entropy for a $k$-SAT instance, based on a discretized form of the von Neumann entropy [Kopp et al. 2007]:

$$
V N(x, p, q)=-\frac{1}{n} \sum_{i=1}^{n} p\left(x_{i}\right) \log \left[q\left(x_{i}\right) p\left(x_{i}\right)\right]
$$


Table II. Dictionary translating entropy status of the SAT formula into its structure as function of $\alpha$

\begin{tabular}{cccccc}
\hline Entropy & Particle status & Information & SAT-instance structure & SAT/UNSAT \\
\hline high & disorder & high & many free variables available to satisfy the few clauses of the formula & SAT \\
low & order & low & few free variables available to satisfy the many clauses of the formula & UNSAT \\
\hline
\end{tabular}

When available, a free variable can be set to TRUE or FALSE so as to satisfy the instance. An instance in the SAT or UNSAT phase is satisfiable or unsatisfiable (respectively) with high probability.

where $p$ and $q$ are respectively a true distribution and an approximated distribution of a random discretized variable $x$ with $n$ possible values. In this work, we set $n=$ number of energy levels, and we compute the entropy every time that a new clause is added to the graph. The final entropy is computed after the last clause of the $k$-SAT instance is added to the final graph. In a BE system, when a new node $\nu$ is added to the graph using the $\mathrm{S} 2 \mathrm{G}$ algorithm, we define:

$$
\begin{aligned}
q_{B E}\left(x_{i}\right)= & \text { number of different physical states } g_{i} \text { on the level } \\
& E_{i} / \text { number of nodes already added to the graph; }
\end{aligned}
$$

Conversely, in a MB system we set

$$
\begin{aligned}
q_{M B}\left(x_{i}\right)= & \text { number of different physical states } g_{i} \text { on the level } \\
& E_{i} / \text { number of nodes already added to the graph; } \\
p_{M B}\left(x_{i}\right)= & \text { probability } p_{i} \text {, computed by } \mathrm{S} 2 \mathrm{G}, \text { that a new node } \\
& \text { is added to the level } E_{i} .
\end{aligned}
$$

In the characterization, the number of nodes of the graph equals the number of available degeneration states, $q$ is the expected distribution followed by the new node when linking to an existing one, and $p$ is the actual distribution, outcome of the actual link established.

In Figure 3 we plot the von Neumann final entropy as function of the clauses-to-variables ratio $\alpha$. The BE systems always have lower entropy than the MB ones, and the entropy behavior is in keeping with the distribution of BEC shown in [Angione et al. 2013]. Hence, the larger is the probability that BEC occurs, the larger is the entropy. Moreover, the plot shows that when $\alpha$ decreases, the entropy increases. This process is associated with an increment of the spatial (geometric) disorder of the particles [Bal'makov 2001]. It follows that the disorder of the particles is related to the satisfiability of the instance (which depends on the value of $\alpha$ ). In this way, we are able to characterize the complexity of certifying the satisfiability of a $k$-SAT instance by computing its entropy (see Table II). We have also performed further analyses, omitted due to lack of space, using the Rényi entropy [Rényi 1961] and the information gain [Niven 2005] to confirm our results.

\subsection{The role of the non-integer out-degree $\theta$}

In [Angione et al. 2013], the parameter $\theta \in] 0 ; 1]$ represents the out-degree increment of the node $v\left(C_{t_{i}}\right)$ linked to the existing node $v\left(C_{t_{j}}\right)$ during the generation of the graph. In particular, the same edge between the new node $v\left(C_{t_{i}}\right)$ and $v\left(C_{t_{j}}\right)$ increases their connectivities $k_{t_{i}}$ and $k_{t_{j}}$ as $k_{t_{i}}=k_{t_{i}}+\theta$ and $k_{t_{j}}=k_{t_{j}}+1$. Making use of the relation $k_{i}=\theta \cdot \operatorname{od}\left(v\left(C_{i}\right)\right)+i d\left(v\left(C_{i}\right)\right)$, where od and $i d$ are the out-degree and in-degree of $v\left(C_{t_{i}}\right)$ respectively, nodes aim to connect to a particular node, which gets richer and richer. Indeed, as incoming links are rewarded more than outgoing links ( 1 and $\theta<1$ respectively), the connectivity of the node that acquires links increases much more than the connectivity of the nodes linking to it. In this work, we aim to show the connection between $\theta$ and the temperature of the system under investigation, namely the $k$-SAT instance.

It is largely known that BEC takes place only in a dilute gas of bosons cooled to temperatures near to the absolute zero [Pethick and Smith 2001]. Therefore, we analyze the system focusing on values of $\theta$ near to zero, in order to prove that for low values of $\theta$ the BEC takes place with more probability than for high values of $\theta$. In order to understand the role played by $\theta$, we analyze 41 


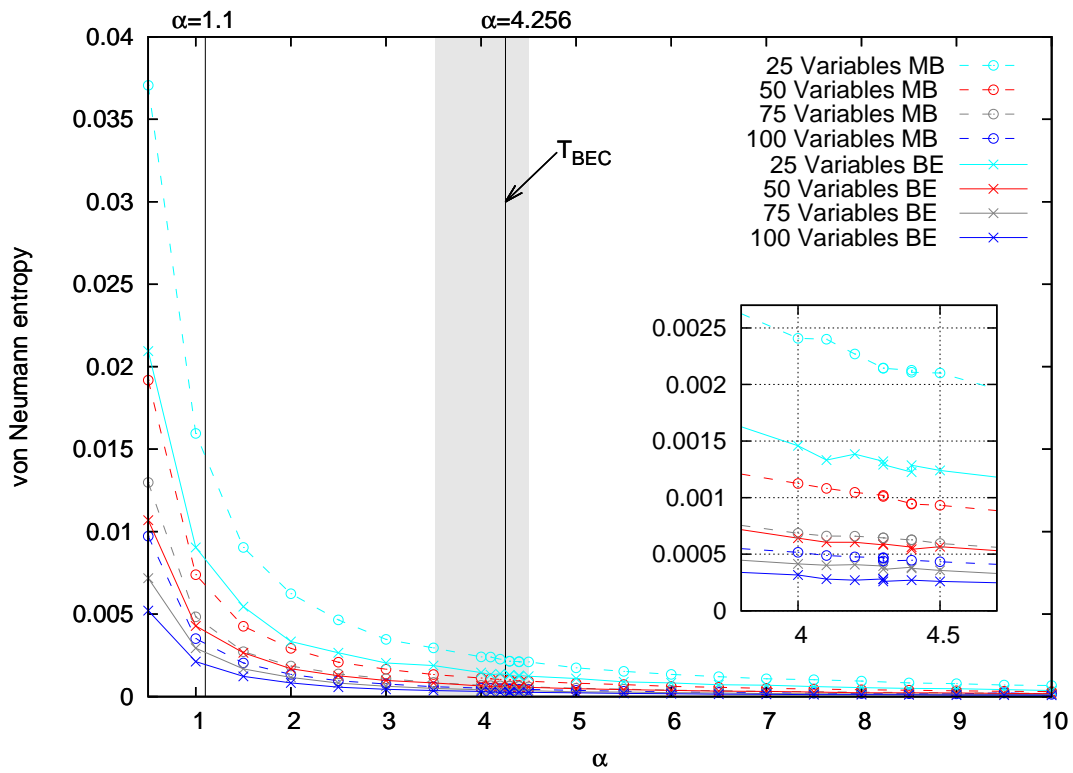

Fig. 3. von Neumann entropy of 3-SAT. The entropy increases as $\alpha$ decreases. Since the entropy represents the spatial disorder of the particles [Bal'makov 2001], in the $k$-SAT problem the physical concept of disorder is related to the complexity of certifying the satisfiability of the instance. The plot shows that BE systems always have lower entropy than MB ones, in agreement with the physical distribution of particles. Indeed, BE systems are often characterized by particles established on the same energy level, which in case of BEC is the lowest available.

different values of $\theta \in] 0,1]$. Figure 4 shows that the probability of BEC increases as $\theta$ decreases. Hence, if $\theta$ approaches zero, the number of BECs increases, suggesting that the parameter $\theta$ in the $\mathrm{S} 2 \mathrm{G}$ algorithm is related to the absolute temperature in the corresponding physical system.

In order to investigate thoroughly the nonlinearity shown by the FW as function of $\theta$, we define $g(\alpha)$ as the third-order polynomial fitting computed with the least-squares approach for each curve plotted in Figure 4. We define the following measure of nonlinearity over an interval $[a, b]$ as $\int_{a}^{b}\left|g^{\prime \prime}(\alpha)\right|$. Using this formula we can compute the amount of variation in the first derivative of $g$, thus quantifying the nonlinearity of $g$ in its domain. Notably, the results shown in Figure 5 allow to identify some clusters of fraction winner plots showing similar nonlinear behavior.

\section{THE MAXWELL-BOLTZMANN CHAINSAT}

In section 3 we focused on the fraction winner related to the $\mathrm{MB}$ and the $\mathrm{BE}$ distributions. The different results obtained by running the $\mathrm{S} 2 \mathrm{G}$ algorithm with these two distributions lead to the creation of the final graphs with different topological structures. That is, the energy assigned by S2G to each clause (i.e., the weight of the clause) changes depending on the distribution being used. In order to study the changes that each distribution involves, here we run the LC version of CHAINSAT [Angione et al. 2013] using the weight provided by the S2G algorithm run with MB and BE distributions. The SAT instances are created through A. van Gelder's generator MKCNF. ${ }^{1}$. The MaxSAT problem consists of determining the truth assignments such that the number of clauses satisfied is maximized [Festa et al. 2007]. In particular, we investigate how the number of satisfied clauses and the number of flips change in each modified version of CHAINSAT (named MB-CHAINSAT and BE-CHAINSAT). Surprisingly, as shown Figure 6, by comparing the percentage of satisfied clauses we can speculate that MB-ChainSAT satisfies the same number of instances as BE-ChainSAT.

\footnotetext{
$1_{\mathrm{ftp} / / \text { dimacs.rutgers.edu/pub/challenge/satisfiability/contributed/UCSC/instances. }}$

We set the generator to obtain both satisfiable and unsatisfiable formulas, so as to obtain a purely uniform random $k$-SAT distribution.
} 

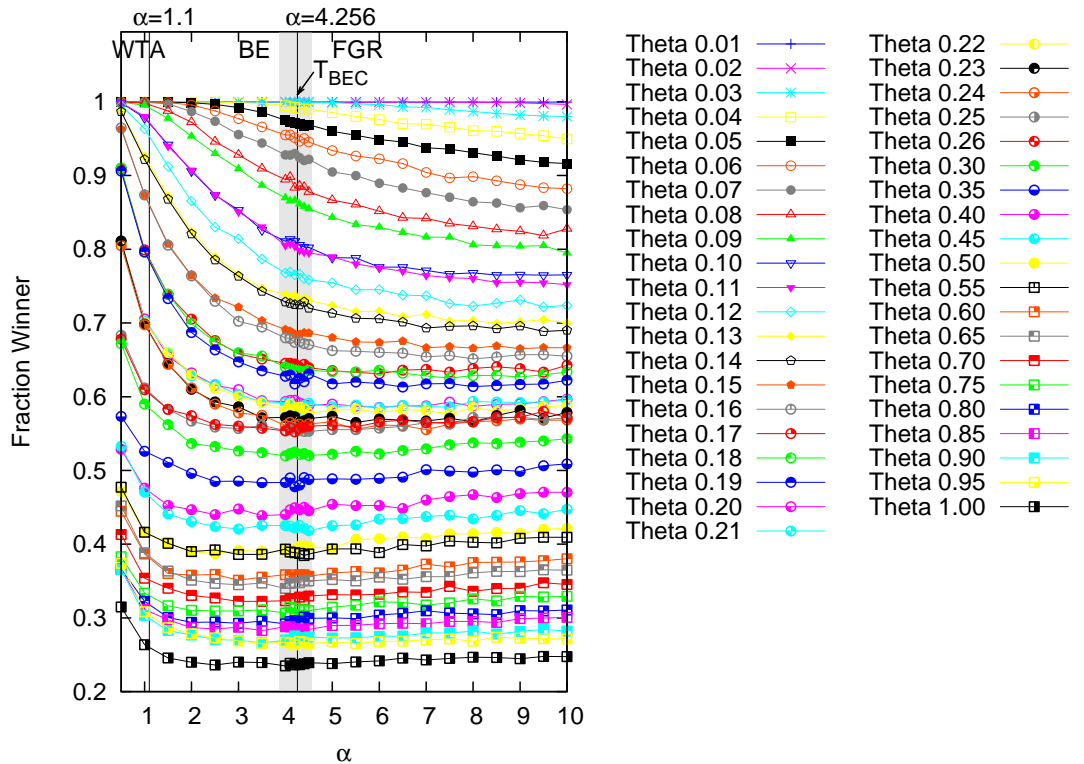

Fig. 4. Fraction Winner of 3-SAT. We report the number of links shared by the winner node against $\alpha$. After a decrease for low values of $\alpha$, the FW is almost linear for high values of $\theta$. For low values of $\theta$, we observe a nonlinear variation, meaning that if $\alpha$ approaches 0 , the number of BEC cases increases with a high derivative. This behavior of $\theta$ is strongly related to the physical property of having a large number of BECs when the absolute temperature approaches 0 .

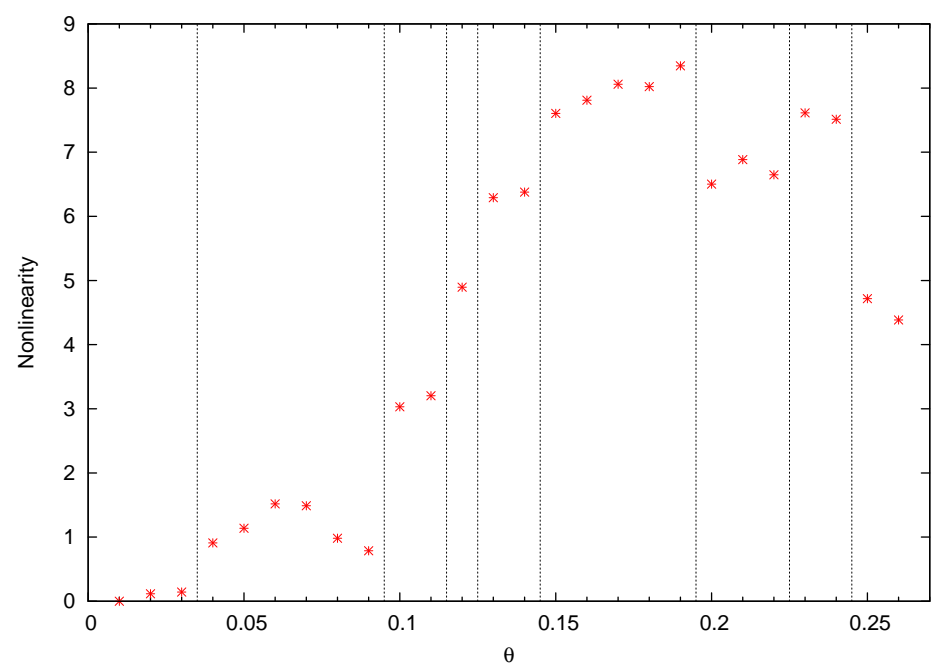

Fig. 5. Nonlinearity of the Fraction Winner as function of $\theta$. The plots of the FW have been clustered according to their nonlinearity, with $\theta \in] 0,0.26]$. When $\theta$ approaches 0 , the $\mathrm{FW}$ exhibits a linear behavior, while the nonlinearity starts from $\theta=0.04$. Interestingly, in $[0.09,0.14]$ there is a large increment of nonlinearity.

We investigate this comparison more thoroughly by analyzing the difference of satisfied clauses for each value of $n$ (number of variables). In particular, we observe that MB-CHAINSAT and BECHAINSAT satisfy approximately the same number of clauses (Table III). We also study the number of flips performed by the two algorithms, representing their computational effort. Figure 7 displays the number of flips (normalized to 1) obtained by running BE-CHAINSAT and MB-CHAINSAT. 


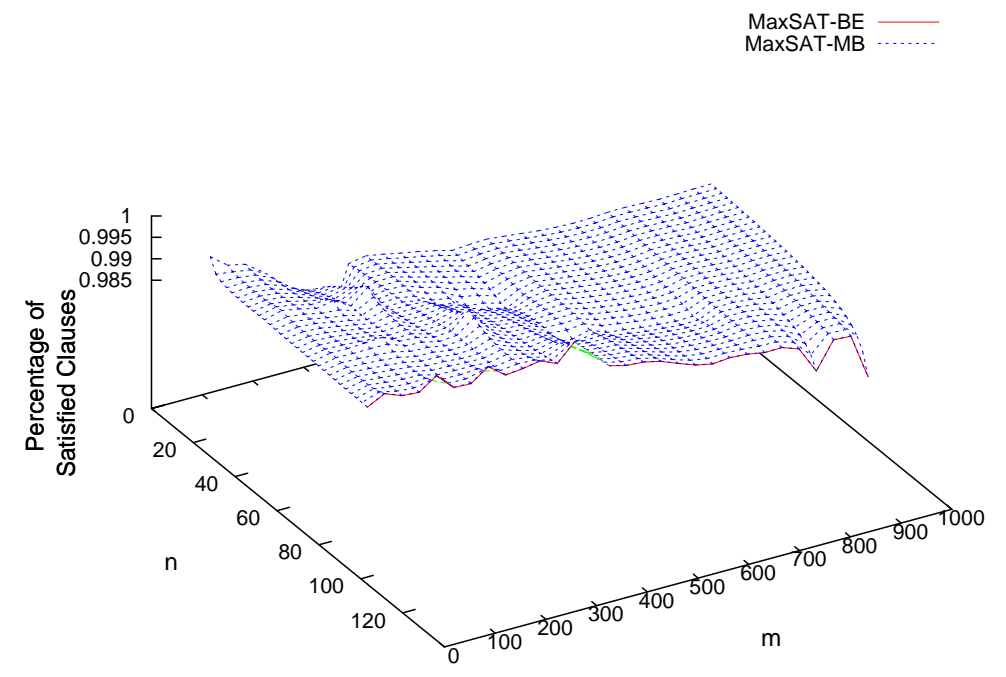

Fig. 6. MaxSAT for LC-ChainSAT. We plot the percentage of clauses satisfied by BE-ChainSAT and MB-ChainSAT as function of the number of clauses $m$ and variables $n$, by using the Linked-Clauses version (LC). Although MB-ChainSAT seems to solve a higher percentage of clauses than BE-ChainSAT, the detailed analysis in Table III highlights that they solve the same number of instances for every value of $n$.

Table III. Summary of LC SAT solvers performance

\begin{tabular}{ccccccc}
\hline & \multicolumn{2}{c}{ LC-Maxwell Boltzmann } & \multicolumn{2}{c}{ LC-Bose Einstein } & \multicolumn{2}{c}{ ChainSAT } \\
$n$ & MaxSAT & Flips & MaxSAT & Flips & MaxSAT & Flips \\
\hline 25 & 0.8567 & 1185219.00 & 0.8567 & 1187645.11 & 0.8567 & $\mathbf{1 1 7 6 7 5 5 . 9 2}$ \\
50 & 0.8580 & 1200317.11 & 0.8580 & $\mathbf{1 1 5 9 2 8 1 . 6 8}$ & 0.8580 & 1165854.05 \\
75 & 0.8587 & 1228486.09 & 0.8587 & $\mathbf{1 2 1 9 5 2 0 . 0 8}$ & 0.8587 & 1231789.81 \\
100 & 0.8590 & 1280600.65 & 0.8590 & $\mathbf{1 2 8 0 0 4 2 . 7 5}$ & 0.8590 & 1296716.23 \\
\hline Average & 0.8581 & 1223655.71 & 0.8581 & $\mathbf{1 2 1 1 6 2 2 . 3 4}$ & 0.8581 & 1217779.00 \\
\hline
\end{tabular}

For each value of $n$ (number of variables), we report the percentage of satisfied clauses and the number of flips performed by MB-CHAINSAT, BE-CHAINSAT and CHAINSAT, using the LC version. All the algorithms ensure the same percentage of satisfied clauses. In terms of flips, CHAINSAT outperforms MB-CHAINSAT and BEChAINSAT only for $n=25$. When $n>25$, BE-CHAINSAT outperforms MB-ChAINSAT and ChAINSAT, as it solves the same percentage of clauses with less flips.

MB-CHAINSAT requires a higher computational effort than BE-CHAINSAT, except for $n=25$. A detailed analysis of the number of flips is shown in Table III.

The average number of flips used for solving an instance with MB-CHAINSAT is 1223655.71 against 1211622.34 with BE-CHAINSAT. Therefore, on average, BE-CHAINSAT needs a lower computational effort than MB-CHAINSAT. This is due to the structure of the BE-graphs, with a fraction winner higher than MB-graphs (as shown in Section 3): a node that shares more links enables to find quickly a truth assignment for the instance, performing less flips. Therefore, if we consider graphs with $n>25$ and our LC version of CHAINSAT, BE-CHAINSAT performs better than MB-CHAINSAT, as it solves the same number of instances performing less flips.

Conversely, when using the NLC version of CHAINSAT [Angione et al. 2013], MB-CHAINSAT generally outperforms BE-CHAINSAT in terms of satisfied clauses, but when $m$ approximates 400, BE-CHAINSAT satisfies more clauses than MB-CHAINSAT (Figure 8). We investigate this comparison more thoroughly by analyzing the difference of satisfied clauses for each value of $n$ (number 


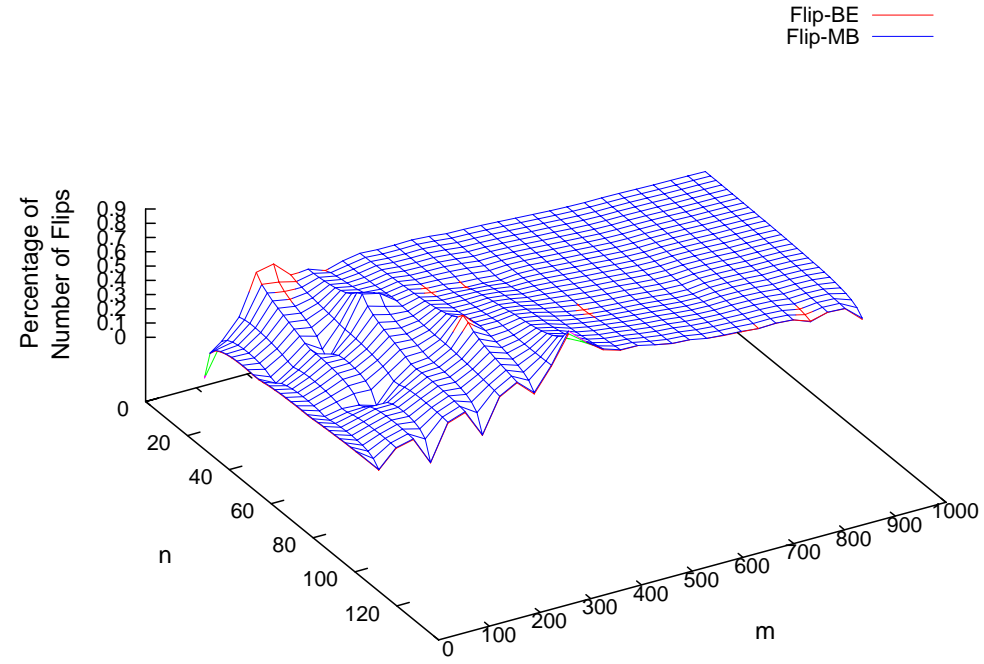

Fig. 7. Computational Effort of LC-CHAINSAT. We plot the number of flips (normalized to 1) performed by BECHAINSAT and MB-CHAINSAT, using the LC version. BE-CHAINSAT employs less flips than MB-CHAINSAT, therefore requiring a lower computational effort. This highlights the importance of the topological structure of a graph. Since with the $\mathrm{BE}$ distribution the graph has a FW higher than the graph built with the MB distribution, there exists a node that, sharing a large number of links, allows to find a solution performing less flips.

Table IV. Summary of NLC SAT solvers performance

\begin{tabular}{ccccccc}
\hline & \multicolumn{2}{c}{ NLC-Maxwell Boltzmann } & \multicolumn{2}{c}{ NLC-Bose Einstein } & \multicolumn{2}{c}{ ChainSAT } \\
$n$ & MaxSAT & Flips & MaxSAT & Flips & MaxSAT & Flips \\
\hline 25 & 0.8567 & $\mathbf{1 1 7 5 2 1 9 . 0 3}$ & 0.8563 & 1177645.11 & 0.8567 & 1176755.92 \\
50 & 0.8577 & $\mathbf{1 1 6 2 9 4 9 . 6 3}$ & 0.8580 & 1166531.26 & 0.8580 & 1165854.05 \\
75 & 0.8587 & $\mathbf{1 2 1 1 3 7 3 . 5 6}$ & 0.8587 & 1229056.62 & 0.8587 & 1231789.81 \\
100 & 0.8590 & $\mathbf{1 2 6 9 2 6 8 . 2 4}$ & 0.8587 & 1275695.48 & 0.8590 & 1296716.23 \\
\hline Average & 0.8580 & $\mathbf{1 2 0 4 7 0 2 . 6 2}$ & 0.8579 & 1212232.12 & 0.8581 & 1217779.00 \\
\hline
\end{tabular}

For each value of $n$ (number of variables), we report the percentage of clauses satisfied and the number of flips performed by MB-CHAINSAT, BE-CHAINSAT and CHAINSAT by using the NLC version. As regards flips, MBCHAINSAT outperforms the other algorithms for every value of $n$.

of variables). The results are shown in Table IV. Finally, in Figure 9 we show that in the NLC case MB-CHAINSAT is computationally less expensive than BE-CHAINSAT.

In conclusion, for the LC version, the BE distribution (namely, BE-CHAINSAT) always outperforms MB-CHAINSAT and CHAINSAT except for $n=25$, as it solves the same percentage of clauses with less flips. Conversely, for the NLC version and in terms of computational effort, the MB distribution (namely, MB-CHAINSAT), outperforms the other algorithms for every value of $n$.

\section{THE MAXWELL-BOLTZMANN PROBSAT AND THE BOSE-EINSTEIN PROBSAT}

We have also analyzed the topological structure of the BE and MB graphs by introducing two new versions of the PROBSAT algorithm. PROBSAT, winner of the Random SAT track from the SAT Competition 2013, is a stochastic local search SAT solver based on probability distributions. Since the algorithm uses a random selection of unsatisfied clauses, we modified this criterion introducing the clauses' energy provided by the S2G algorithm. In particular, we replaced the random choice 


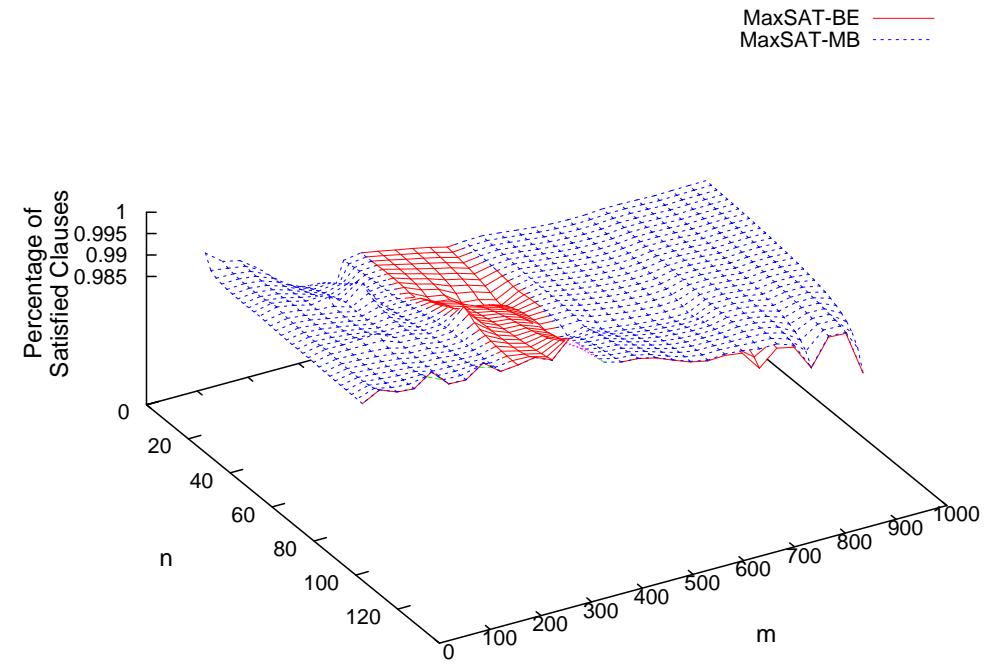

Fig. 8. MaxSAT for NLC-CHAINSAT. We plot the percentage of clauses satisfied by BE-CHAINSAT and MBCHAINSAT as function of the number of clauses $m$ and variables $n$, by using the Not-Linked-Clauses versions (NLC). MB-CHAINSAT seems to solve a higher percentage of clauses than BE-CHAINSAT. Actually, MB-CHAINSAT outperforms BE-CHAINSAT only for few values of $\alpha$. The two algorithms solve exactly the same number of instances for $n=75$. (A more detailed analysis is shown in Table IV.)

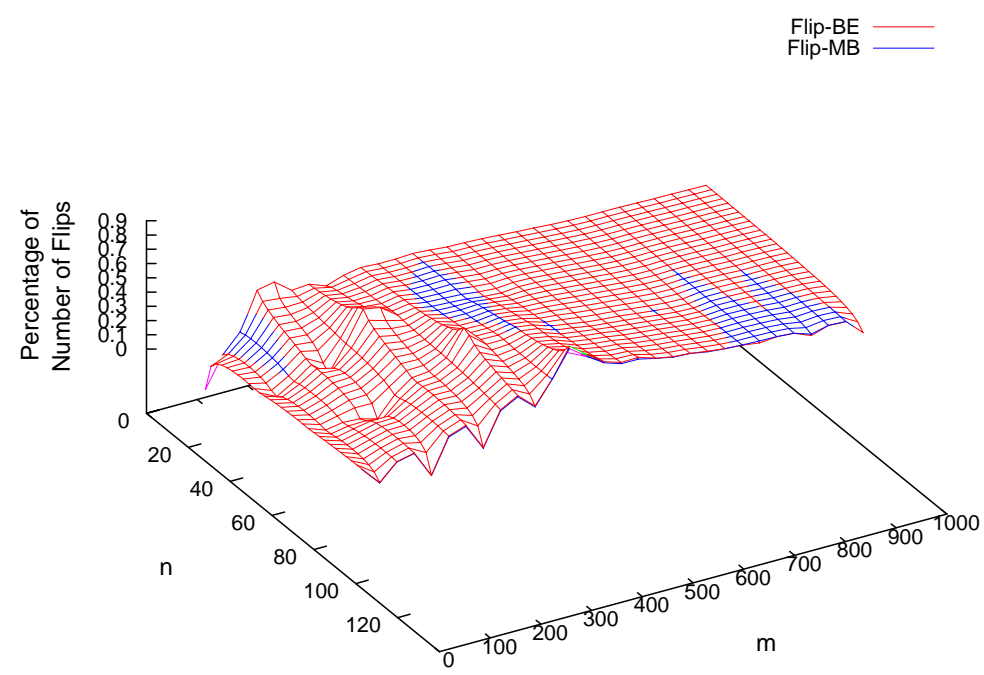

Fig. 9. Computational Effort of NLC-CHAINSAT. We plot the number of flips (normalized to 1) performed by the two algorithms by using the NLC version. BE-CHAINSAT employs more flips than MB-CHAINSAT, thus requiring a higher computational effort. 
Table V. Summary of PROBSAT solvers performance

\begin{tabular}{ccccccc}
\hline & \multicolumn{2}{c}{ Maxwell Boltzmann-ProbSAT } & \multicolumn{2}{c}{ Bose Einstein-ProbSAT } & \multicolumn{2}{c}{ ProbSAT } \\
$n$ & MaxSAT & Flips & MaxSAT & Flips & MaxSAT & Flips \\
\hline 25 & $\mathbf{0 . 9 5 3 0}$ & 550801.42 & 0.9526 & 550803.49 & 0.9526 & 550797.85 \\
50 & $\mathbf{0 . 9 0 7 9}$ & 592512.60 & 0.9078 & 592525.46 & 0.9071 & 592498.46 \\
75 & 0.8653 & $\mathbf{6 0 3 6 7 6 . 0 2}$ & $\mathbf{0 . 8 6 6 5}$ & 603680.32 & 0.8645 & 603711.44 \\
100 & 0.8217 & 610411.94 & 0.8202 & 610441.18 & 0.8227 & 610311.33 \\
\hline Average & $\mathbf{0 . 8 8 7 0}$ & 589350.49 & 0.8868 & 589362.61 & 0.8867 & 589329.77 \\
\hline
\end{tabular}

For each value of $n$ (number of variables), we report the percentage of clauses satisfied and the number of flips performed by MB-ProbSAT, BE-ProbSAT and ProbSAT. As regards the percentage of clauses satisfied, MB-ProBSAT and BE-PROBSAT outperform the PROBSAT algorithm for $n=25, n=50$ and $n=75$.

of the unsatisfied clause with an ordered one. By using the same method of the NLC-CHAINSAT [Angione et al. 2013], we selected the clause with the lowest energy among the unsatisfied clauses. Hence, we obtained two versions of ProbSAT: MB-ProbSAT and BE-ProbSAT, which take into account the clauses' energy provided by the S2G algorithm run with MB and BE distributions respectively. As done before for the CHAINSAT algorithm, if all the clauses in the set of unsatisfied clauses have been already chosen once, we reset the original random choice to prevent that the algorithm always analyzes the same clauses.

We tested the algorithms on the same set of instances previously used for the ChainSAT algorithm and we ran the PROBSAT with maxTries $=1$ and maxFlips $=10^{6}$. In Table V, we present a comparison between the three different versions of ProBSAT (MB-ProBSAT, BE-PROBSAT and ProbSAT). Both MB-ProbSAT and BE-ProbSAT satisfy more clauses than the original PROBSAT, except for $n=100$. In terms of flips, the two modified versions require a slightly higher computational effort than PROBSAT. This implies that choosing the clauses with the lowest energy provides a useful tool to maximize the number of satisfied clauses, confirming the hypothesis that the clauses with the lowest energy play an important role in the performance of the SAT solvers.

\section{DISCUSSION AND CONCLUSIONS}

In this research work, we have proposed a statistical-physical characterization for the Satisfiability problem. Starting from the S2G algorithm [Angione et al. 2013], we have developed a new algorithm able to translate a SAT instance into a graph by using the $\mathrm{BE}$ (quantum) or the MB (classical) statistical distributions. The phase diagram of the graph provided by the algorithm shows evidence of condensation as the clauses-to-variables ratio decreases. Furthermore, we have carried out a systematic study to employ the characterization in the well-known CHAINSAT [Alava et al. 2008] and PROBSAT [Balint and Fröhlich 2010] solvers, without requiring a priori investigation of their solutions. The fitness-based sorting provided by our algorithm allows to enhance both solvers.

In order to investigate the role of the quantum physics in our algorithm, we have cross-compared the behavior of our algorithm when using classical or quantum physics. From the phase diagrams, it is evident that graphs are more likely to undergo BEC if they are generated through the algorithm based on quantum physics, while the fit-get-rich behavior is more likely with the algorithm based on classical physics. Moreover, when using our quantum and classical algorithms to drive the CHAINSAT and PROBSAT solvers, BE-CHAINSAT is computationally less or more expensive than MB-CHAINSAT, in terms of flips performed, depending on the CHAINSAT version used (LC or NLC), while MB-PROBSAT outperforms BE-PROBSAT in terms of flips.

Notably, we have also defined the entropy of a SAT instance according to the information gained during the generation of its graph through S2G. We have obtained that a high entropy is associated with high disorder, and with the SAT phase. An instance with high entropy has a large number of free variables that can be assigned a convenient truth value to satisfy the SAT instance. Furthermore, from a statistical-physical standpoint, we have shown that the temperature of the SAT problem is mapped by the parameter representing the out-degree assigned dynamically to the nodes of the 
graph associated with the instance. These findings highlight the emergence of a comprehensive characterization of the $k$-SAT problem using the classical and quantum particle distributions of statistical physics.

\section{Acknowledgements}

The authors would like to thank Prof. A. Majorana for the seminal discussion on the classical and quantum particle distributions.

\section{REFERENCES}

D. Achlioptas and F. Ricci-Tersenghi. 2009. Random formulas have frozen variables. SIAM J. Comput. 39, 1 (2009), 260280.

M. Alava, J. Ardelius, E. Aurell, P. Kaski, S. Krishnamurthy, P. Orponen, and S. Seitz. 2008. Circumspect descent prevails in solving random constraint satisfaction problems. Proceedings of the National Academy of Sciences 105, 40 (2008), 15253.

C. Angione, A. Occhipinti, G. Stracquadanio, and G. Nicosia. 2013. Bose-Einstein Condensation in Satisfiability Problems. European Journal of Operational Research 227, 1 (2013), 44-54.

Adrian Balint and Andreas Fröhlich. 2010. Improving stochastic local search for SAT with a new probability distribution. In Theory and Applications of Satisfiability Testing-SAT 2010. Springer, 10-15.

M. D. Bal'makov. 2001. Entropy and disorder. Glass physics and chemistry 27, 4 (2001), 287-297.

G. Bianconi and A.L. Barabási. 2001. Bose-Einstein condensation in complex networks. Physical Review Letters 86, 24 (2001), 5632-5635.

E. Clarke, A. Gupta, H. Jain, and H. Veith. 2008. Model checking: Back and forth between hardware and software. Verified Software: Theories, Tools, Experiments (2008), 251-255.

T.H. Cormen, C.E. Leiserson, R.L. Rivest, and C. Stein. 2001. Introduction to algorithms. MIT press.

Dingzhu Du, Jun Gu, Panos M Pardalos, and others. 1997. Satisfiability problem: theory and applications: DIMACS Workshop, March 11-13, 1996. Vol. 35. American Mathematical Soc.

P. Festa, P.M. Pardalos, L.S. Pitsoulis, and M.G.C. Resende. 2007. GRASP with path relinking for the weighted MAXSAT problem. Journal of Experimental Algorithmics (JEA) 11, Article 2.4 (2007).

E. Grégoire, B. Mazure, and C. Piette. 2009. Using local search to find MSSes and MUSes. European Journal of Operational Research 199, 3 (2009), 640-646.

Y. Hamadi, S. Jabbour, and L. Sais. 2009. Control-based clause sharing in parallel SAT solving. In Proceedings of the 21st International Joint Conference on Artificial Intelligence. Morgan Kaufmann Publishers Inc., 499-504.

H. Kautz and B. Selman. 1999. Unifying SAT-based and graph-based planning. In International Joint Conference on Artificial Intelligence, Vol. 16. Lawrence Erlbaum associates LTD, 318-325.

A. Kopp, X. Jia, and S. Chakravarty. 2007. Replacing energy by von Neumann entropy in quantum phase transitions. Annals of Physics 322, 6 (2007), 1466-1476.

M. Mézard and A. Montanari. 2007. Constraint satisfaction networks in Physics and Computation. Clarendon Press, Oxford 1,9 (2007), 11.

M. Mézard and T. Mora. 2009. Constraint satisfaction problems and neural networks: a statistical physics perspective. Journal of Physiology-Paris 103, 1 (2009), 107-113.

M. Mézard, G. Parisi, and R. Zecchina. 2002. Analytic and algorithmic solution of random satisfiability problems. Science 297, 5582 (2002), 812-815.

D. Mitchell, B. Selman, and H. Levesque. 1992. Hard and easy distributions of SAT problems. In Proceedings of the National Conference on Artificial Intelligence. AAAI Press/MIT Press, 459-465.

R. Monasson, R. Zecchina, S. Kirkpatrick, B. Selman, and L. Troyansky. 1999. Determining computational complexity from characteristic phase transitions. Nature 400, 6740 (1999), 133-137.

R.K. Niven. 2005. Exact Maxwell-Boltzmann, Bose-Einstein and Fermi-Dirac statistics. Physics Letters A 342, 4 (2005), 286-293.

K. Nonobe and T. Ibaraki. 1998. A tabu search approach to the constraint satisfaction problem as a general problem solver. European Journal of Operational Research 106, 2 (1998), 599-623.

R.K. Pathria. 1996. Statistical mechanics. Butterworth-Heinemann.

O. Penrose and L. Onsager. 1956. Bose-Einstein condensation and liquid helium. Physical Review 104, 3 (1956), 576-584.

C.J. Pethick and H. Smith. 2001. Bose-Einstein condensation in dilute gases. Cambridge university press.

A. Rényi. 1961. On measures of entropy and information. In Fourth Berkeley Symposium on Mathematical Statistics and Probability. 547-561. 
T. Richardson and R. Urbanke. 2008. Modern coding theory. Cambridge University Press.

SAT-competition. 2013. http://www.satcompetition.org/. (2013).

I. Spence. 2010. sgen1: A generator of small but difficult satisfiability benchmarks. Journal of Experimental Algorithmics (JEA) 15, Article 1.2 (2010).

H.J. Zimmermann and A. Monfroglio. 1997. Linear programs for constraint satisfaction problems. European Journal of Operational Research 97, 1 (1997), 105-123. 